\title{
Plasma D-dimer may predict poor functional outcomes through systemic complications after aneurysmal subarachnoid hemorrhage
}

\author{
Hitoshi Fukuda, MD, ${ }^{1,2}$ Benjamin Lo, MD, ${ }^{4}$ Yu Yamamoto, MD, ${ }^{1}$ Akira Handa, MD, ${ }^{2}$ \\ Yoshiharu Yamamoto, BS, ${ }^{3}$ Yoshitaka Kurosaki, MD, ${ }^{1}$ and Sen Yamagata, MD'

\begin{abstract}
Departments of ${ }^{1}$ Neurosurgery, ${ }^{2}$ nterventional Neuroradiology, and ${ }^{3} \mathrm{Clinical}$ Research, Kurashiki Central Hospital, Kurashiki, Okayama, Japan; and ${ }^{4}$ Department of Neurosurgery, Montreal Neurological Institute and Hospital, McGill University Health Centre, Montreal, Quebec, Canada
\end{abstract}

\begin{abstract}
OBJECTIVE Plasma D-dimer levels elevate during acute stages of aneurysmal subarachnoid hemorrhage (SAH) and are associated with poor functional outcomes. However, the mechanism in which D-dimer elevation on admission affects functional outcomes remains unknown. The aim of this study is to clarify whether D-dimer levels on admission are correlated with systemic complications after aneurysmal SAH, and to investigate their additive predictive value on conventional risk factors for poor functional outcomes.
\end{abstract}

METHODS A total of 187 patients with aneurysmal SAH were retrospectively analyzed from a single-center, observational cohort database. Correlations of plasma D-dimer levels on admission with patient characteristics, initial presentation, neurological complications, and systemic complications were identified. The authors also evaluated the additive value of $\mathrm{D}$-dimer elevation on admission for poor functional outcomes by comparing predictive models with and without D-dimer.

RESULTS D-dimer elevation on admission was associated with increasing age, female sex, and severity of SAH. Patients with higher D-dimer levels had increased likelihood of nosocomial infections (OR 1.22 [95\% CI 1.07-1.39], p $=0.004$ ), serum sodium disorders (OR 1.11 [95\% Cl 1.01-1.23], $p=0.033)$, and cardiopulmonary complications (OR 1.20 [95\% Cl 1.04-1.37], $p=0.01$ ) on multivariable analysis. D-dimer elevation was an independent risk factor of poor functional outcome (modified Rankin Scale Score 3-6, OR 1.50 [95\% Cl 1.15-1.95], $p=0.003$ ). A novel prediction model with D-dimer had significantly better discrimination ability for poor outcomes than conventional models without D-dimer. CONCLUSIONS Elevated D-dimer levels on admission were independently correlated with systemic complication, and had an additive value for outcome prediction on conventional risk factors after aneurysmal SAH.

https://thejns.org/doi/abs/10.3171/2016.5.JNS16767

KEY WORDS subarachnoid hemorrhage; D-dimer; systemic complications; functional outcome; vascular disorders

$\mathrm{E}$ LEVATED plasma levels of the fibrin degradation product D-dimer predict poor functional outcomes after aneurysmal subarachnoid hemorrhage (SAH) ${ }^{8,9,14,16,20}$ Yet the precise mechanisms in which D-dimer elevation is associated with poor functional outcomes in aneurysmal SAH remain unclear. D-dimer elevation may reflect hemostatic and coagulopathic disturbances occurring in multiple organ systems. In this article, we investigate whether plasma $\mathrm{D}$-dimer levels at admission are correlated with systemic complications after aneurysmal
SAH and assess discriminative performance of a novel outcome prediction model comprising conventional risk factors plus D-dimer.

\section{Methods}

The study is reported based on criteria from the STROBE (Strengthening the Reporting of Observational Study in Epidemiology) statement. ${ }^{27}$ The study protocol was approved by the Kurashiki Central Hospital Research

ABBREVIATIONS DCI = delayed cerebral infarction; DIND = delayed ischemic neurological deficit; ICH = intracerebral hemorrhage; IDI = integrated discrimination improvement; IVH = intraventricular hemorrhage; $\mathrm{mRS}$ = modified Rankin Scale; NRI = net reclassification improvement; SAH = subarachnoid hemorrhage; WFNS = World Federation of Neurosurgical Societies.

SUBMITTED March 26, 2016. ACCEPTED May 27, 2016.

INCLUDE WHEN CITING Published online August 12, 2016; DOI: 10.3171/2016.5.JNS16767. 
Ethics Committee, and waiver of consent was sought and obtained for this cohort study with no unique patient identifiers.

\section{Patient Selection, Study Design, and Clinical Evaluation}

This is a retrospective cohort study comprising 187 consecutive patients with aneurysmal SAH, who were treated within 72 hours after symptom onset between January 2012 and June 2015 at our institution. Patients were excluded from surgical or endovascular intervention if they had obvious evidence of neurologically irreversible brainstem injury or their next of kin indicated limits on therapeutic interventions.

Age, sex, hypertension, Type 2 diabetes status, preoperative antiplatelet or anticoagulant use, aneurysm location (anterior circulation or posterior), thick subarachnoid clot (short axis $\geq 4 \mathrm{~mm}$ ), intracerebral hemorrhage (ICH), intraventricular hemorrhage (IVH), and initial neurological state classified with World Federation of Neurosurgical Societies (WFNS) grade, ${ }^{5}$ were analyzed for any correlation with plasma D-dimer level on admission.

Subsequently, neurological complications including seizure, delayed ischemic neurological deficit (DIND), delayed cerebral infarction (DCI), and chronic hydrocephalus, and systemic complications including nosocomial infections, fever $\left(>38^{\circ} \mathrm{C}\right)$ on Day 7 , serum sodium disorders, and cardiopulmonary complications, were investigated to assess the contribution of plasma D-dimer level on these factors. Five patients who died early (within 7 days) were excluded from these complication-related analyses. DIND was defined as clinical deterioration occurring between Days 4 and 14 after SAH, including confusion or decline in level of consciousness, or focal deficits not clinically or radiographically attributable to other causes. DCI was defined as newly developed low-density areas on postoperative CT scans as a result of DIND. Infections included clinical and microbiological confirmation of meningitis, pneumonia, bacteremia, colitis, wound infection, and urinary tract infection at any point during hospitalization, treated with antimicrobials? . Serum sodium was measured every $2-3$ days, and hyponatremia and hypernatremia with serum sodium concentrations less than $135 \mathrm{mmol} / \mathrm{L}$ and more than $150 \mathrm{mmol} / \mathrm{L}$, respectively, were defined as serum sodium disorders. ${ }^{23}$ Cardiopulmonary complications included clinically significant congestive heart failure, Takotsubo cardiomyopathy, ${ }_{15}$ and pulmonary edema, which were diagnosed based on the results of chest radiographs, electrocardiograms, and echocardiograms and were treated with diuretics, inotropes, or steroids, as directed by a cardiologist.

Patients' functional outcome 3 months after onset was determined using the modified Rankin Scale (mRS), as assessed by a physician (S.Y.) blinded to patients' D-dimer levels on admission. Functional outcomes were dichotomized into good (mRS Scores 0-2) and poor (mRS Scores 3-6). The relationship between plasma D-dimer levels and poor functional outcome was analyzed, along with the other covariates. Also, the additive predictive value of $\mathrm{D}$-dimer for poor outcome was investigated by comparing prognostic models with and without D-dimer.

\section{Treatment Protocol}

Multidisciplinary discussions were conducted to determine treatment modality, surgical clipping or endovascular coiling, of ruptured aneurysms. In general, aneurysms in younger patients, those of very small sizes, or those with wide necks or aberrant branches, were treated with surgical clipping. Microsurgery was also indicated for patients with increased intracranial pressure requiring hematoma evacuation and hemicraniectomy. Endovascular coiling was selected for aneurysms in older patients with comorbidities, posterior circulation aneurysms, or aneurysms with small necks.

Vasospasm was prevented by a continuous cisternal irrigation protocol with milrinone, a bipyridine methyl carbonitrile analog of amrinone with both inotropic and vasodilating properties. ${ }^{1,25}$ When angiographic vasospasm was observed on 3D CT angiography, which was performed every 3-4 days, the irrigation solution consisted of lactated Ringer solution, urokinase $(120 \mathrm{IU} / \mathrm{ml})$, ascorbic acid $(3.5 \mathrm{mg} / \mathrm{ml})$, and milrinone $(3.6 \mu \mathrm{g} / \mathrm{ml})$, and was continuously infused between the inlet and outlet tubes inserted in the subarachnoid space until Day 14 of SAH. Intravenous fasudil chloride was also administered during this period. Percutaneous transluminal angioplasty for vasospasm was performed when symptomatic vasospasm was caused by severe stenosis of major intracranial arteries.

\section{D-Dimer Measurement}

Blood samples were obtained in all 187 patients within 1 hour after admission. Free-flowing blood was collected into polyethylene terephthalate tubes containing sodium citrate $(3.2 \%, 0.11 \mathrm{~mol} / \mathrm{L})$ as the anticoagulant at a ratio of 1:9 (anticoagulant/blood ratio). The samples were centrifuged for 10 minutes at $1670 \mathrm{~g}\left(22^{\circ} \mathrm{C}\right)$ to separate the plasma. D-dimers were measured with a quantitative photometric latex immunoassay (Tinaquant; Roche Diagnostics $\mathrm{GmbH}$ ). According to the manufacturer's instruction and the institutional agreement, the value of $\mathrm{D}$-dimer $\geq 1.0$ $\mu \mathrm{g} / \mathrm{ml}$ was defined as elevated.

\section{Data Analysis}

Quantitative variables are expressed as mean \pm standard deviation or median value and interquartile range (IQR 25th-75th percentile) as appropriate. The chi-square test was used to evaluate covariates for binary categorical dependent variables. Normality of data was evaluated using the Shapiro-Wilk test. Nonnormally distributed continuous variables were analyzed using the Mann-Whitney U-test. Significant correlation of D-dimer with clinical courses or poor functional outcome was evaluated using univariate logistic regression. Only variables with $\mathrm{p}<0.10$ in the univariate analysis were included in the multivariable logistic regression model-building process. Models were built using forward/backward stepwise logistic regression with variables entered into the model and removed at a 0.10 significance level. The odds ratios and 95\% confidence intervals were also determined in the logistic regression analysis.

To compare the discrimination of poor functional outcome between the models adjusted for known risk factors 
TABLE 1. Characteristics of 187 SAH patients according to plasma D-dimer levels on admission*

\begin{tabular}{lccc}
\hline \multirow{2}{*}{\multicolumn{1}{c}{ Variable }} & \multicolumn{2}{c}{ D-Dimer Levels } & \\
\cline { 2 - 3 } & $\begin{array}{c}\text { Elevated } \\
(\mathrm{n}=124)\end{array}$ & $\begin{array}{c}\text { Normal } \\
(\mathrm{n}=63)\end{array}$ & $\begin{array}{c}\mathrm{p} \\
\text { Value }\end{array}$ \\
\hline Mean age \pm SD in yrs & $68.0 \pm 13.3$ & $56.9 \pm 13.6$ & $<0.001$ \\
\hline Women & $107(86.3)$ & $43(68.3)$ & $\mathbf{0 . 0 0 3}$ \\
\hline Hypertension & $54(43.5)$ & $29(46.0)$ & 0.74 \\
\hline Type 2 diabetes & $9(7.3)$ & $6(9.5)$ & 0.59 \\
\hline Antiplatelet/anticoagulant use & $9(7.3)$ & $3(4.8)$ & 0.51 \\
\hline WFNS Grades IV-V & $57(46.0)$ & $8(12.7)$ & $<0.001$ \\
\hline Posterior circulation & $21(16.9)$ & $12(19.0)$ & 0.72 \\
\hline Thick hematoma & $75(60.5)$ & $21(33.3)$ & $<0.001$ \\
\hline ICH & $21(16.9)$ & $10(15.9)$ & 0.85 \\
\hline IVH & $62(50.0)$ & $20(31.7)$ & 0.017 \\
\hline
\end{tabular}

* Values are presented as the number of patients (\%) unless indicated otherwise. Boldface type indicates statistical significance by univariate analysis ( $p$ $<0.05$ )

with and without D-dimer, $\mathrm{C}$ statistics analogous to the area under the receiver operating curve were estimated. ${ }^{21}$ The statistical significance of differences was compared using the method of DeLong et al. ${ }^{4}$ The increased discriminatory value of plasma D-dimer levels was further examined by the net reclassification improvement (NRI) and integrated discrimination improvement (IDI). ${ }^{22}$ To create the cross-tabulation, we classified the probability of the poor functional outcome into 4 categories of $<23 \%$, $23 \%$ to $48 \%, 48 \%$ to $76 \%$, and $>76 \%$, referring to the median value and the quartiles of the predicted probability of all patients. The continuous NRI value evaluates changes in estimated prediction probabilities between different models. The IDI considers differences in discrimination slopes between different models, where the discrimination slope was defined as the difference between the mean of the estimated prediction probabilities taken as continuous variables for individuals with events and the corresponding mean for those without events. Probability values <
0.05 were considered statistically significant. Statistical analyses were conducted using R software (version 3.1.1, R Foundation for Statistical Computing; http://www.Rproject.org).

\section{Results \\ D-Dimer and Baseline Characteristics}

Baseline characteristics of 187 SAH patients according to plasma D-dimer levels on admission are summarized in Table 1. D-dimer was elevated ( $\geq 1.0 \mu \mathrm{g} / \mathrm{ml})$ in 124 of 187 patients with SAH $(66.3 \%)$. Increasing age $(\mathrm{p}<0.001)$ and female sex $(p=0.003)$ were significantly associated with elevated plasma D-dimer level. Patients with poorer initial neurological state (WFNS Grades IV-V) more likely presented with D-dimer elevation $(\mathrm{p}<0.001)$. In terms of initial head CT findings, thick hematoma $(\mathrm{p}<0.001)$ and presence of IVH $(p=0.017)$ were significantly correlated with D-dimer elevation.

\section{D-Dimer and Complications During Clinical Courses}

Table 2 shows the results of univariate logistic regression analysis for contribution of D-dimer elevations to complications during patients' clinical courses. Five patients who died within 7 days after onset were excluded. Among neurological complications, chronic hydrocephalus (OR 1.15 [95\% CI 1.03-1.27], $p=0.01$ ) was significantly associated with elevated D-dimer level. Associations of DIND ( $p=0.065)$ and DCI $(p=0.078)$ were marginally significant. Among systemic complications, nosocomial infection was observed in 65 cases $(35.7 \%)$ and categorized into pneumonia (21 cases), meningitis (15 cases), urinary tract infection (20 cases), bacteremia (5 cases), colitis (3 cases), and wound infection (1 case). Serum sodium disorder was observed in 69 cases (37.9\%), with hyponatremia (54 cases) and hypernatremia (15 cases). Cardiopulmonary complications were observed in 17 cases (9.3\%); congestive heart failure (5 cases), left ventricular dysfunction by Takotsubo cardiomyopathy ( 9 cases), and pulmonary edema with relatively normal cardiac function (3 cases) were diagnosed by cardiologists. All systemic complications that were investigated, including infection (OR 1.33

TABLE 2. Univariate analysis of contribution of plasma D-dimer level to complications after SAH*

\begin{tabular}{llrr}
\hline \multicolumn{1}{c}{ Complications } & Median Plasma D-Dimer (IQR) & OR $(95 \% \mathrm{Cl})$ & $\mathrm{p} \mathrm{Value}$ \\
\hline Neurological complications & & & \\
\hline Seizure $(\mathrm{n}=14)$ & $1.65(0.7-3.4)$ & $1.07(0.93-1.24)$ & 0.34 \\
\hline DIND $(\mathrm{n}=24)$ & $2.1(0.75-5.2)$ & $1.11(0.99-1.25)$ & 0.065 \\
\hline $\mathrm{DCl}(\mathrm{n}=12)$ & $3.25(2.15-5.4)$ & $1.13(0.99-1.31)$ & 0.078 \\
\hline Chronic hydrocephalus $(\mathrm{n}=38)$ & $2.75(1.4-5.1)$ & $1.15(1.03-1.27)$ & $\mathbf{0 . 0 1}$ \\
\hline Systemic complications & & & \\
\hline Nosocomial infection $(\mathrm{n}=65)$ & $3.3(1.6-5.3)$ & $1.33(1.16-1.53)$ & $<0.001$ \\
\hline Fever on Day 7 ( $=57)$ & $2.6(1.5-4.7)$ & $1.21(1.08-1.35)$ & $\mathbf{0 . 0 0 1}$ \\
\hline Serum sodium disorder $(\mathrm{n}=69)$ & $2.0(0.7-4.05)$ & $1.11(1.00-1.22)$ & $\mathbf{0 . 0 4 3}$ \\
\hline Cardiopulmonary complications $(\mathrm{n}=17)$ & $5.0(3.3-7.8)$ & $1.28(1.13-1.45)$ & $<0.001$ \\
\hline
\end{tabular}

* Five patients who died within 7 days after the onset are excluded. Boldface type indicates statistical significance $(p<0.05)$ by univariate analysis. Italic type indicates marginally significant variables $(p<0.10)$. 
TABLE 3. Multivariable analysis for contribution of plasma D-dimer level to complications*

\begin{tabular}{lll}
\hline \multicolumn{1}{c}{ Complications $\dagger$} & Adjusted OR $(95 \% \mathrm{Cl})$ & p Value \\
\hline Neurological complications & & \\
\hline DIND & $1.09(0.96-1.24)$ & 0.19 \\
\hline $\mathrm{DCl}$ & $1.02(0.85-1.22)$ & 0.84 \\
\hline Chronic hydrocephalus & $1.02(0.90-1.16)$ & 0.72 \\
\hline Systemic complications & & \\
\hline Nosocomial infection & $1.22(1.07-1.39)$ & $\mathbf{0 . 0 0 4}$ \\
\hline Fever on Day 7 & $1.02(0.96-1.22)$ & 0.21 \\
\hline Serum sodium disorder & $1.11(1.01-1.23)$ & $\mathbf{0 . 0 3 3}$ \\
\hline Cardiopulmonary complications & $1.20(1.04-1.37)$ & $\mathbf{0 . 0 1}$ \\
\hline
\end{tabular}

* Five patients who died within 7 days after the onset are excluded.

$\dagger$ Adjusted for the following covariates. DIND: WFNS Grades IV-V, thick hematoma, ICH. DCl: hypertension, WFNS Grades IV-V, thick hematoma, IVH. Chronic hydrocephalus: age, antiplatelet/anticoagulant use, WFNS Grades IV-V, thick hematoma, IVH. Nosocomial infection: age, WFNS Grades IV-V, thick hematoma. Fever at Day 7: age, WFNS Grades IV-V, coil embolization, thick hematoma, ICH, IVH. Serum sodium disorder: age, Type 2 diabetes, coil embolization. Cardiopulmonary complications: age, WFNS Grades IV-V, coil embolization, thick hematoma, IVH.

$\ddagger$ Boldface type indicates statistical significance $(p<0.05)$ by multivariable analysis.

[95\% CI 1.16-1.53], $\mathrm{p}<0.001$ ), fever on Day 7 (OR 1.21 [95\% CI 1.08-1.35], $\mathrm{p}=0.001$ ), serum sodium disorder (OR 1.11 [95\% CI 1.00-1.22], $\mathrm{p}=0.043$ ), and cardiopulmonary complications (OR 1.28 [95\% CI 1.13-1.45], p < 0.001 ), were significantly correlated with elevated D-dimer level by univariate logistic regression.

Table 3 shows the results of multivariable logistic regression analysis for contribution of elevated D-dimer level to complications. None of the neurological complications were significantly associated with D-dimer elevation, after adjustment for potential confounders, including initial WFNS grade and intracranial clot burden. On the other hand, D-dimer elevation remained a significant risk factor for systemic complications including infection (OR 1.22 [95\% CI 1.07-1.39], $\mathrm{p}=0.004$ ), serum sodium disorder (OR 1.11 [95\% CI 1.01-1.23], $\mathrm{p}=0.033$ ), and cardiopulmonary complications (OR 1.20 [95\% CI 1.04-1.37], $\mathrm{p}=0.01$ ), by multivariable logistic regression analyses.

\section{Additive Value of D-Dimer for Prediction of Poor Functional Outcome}

Table 4 shows the results of univariate logistic regression analysis of factors associated with poor functional outcome (mRS Scores 3-6). Univariate analysis demonstrated that elevated D-dimer levels were significantly associated with poor functional outcome (OR 2.01 [95\% CI $1.55-2.61], p<0.001)$. In multivariable logistic regression analysis, elevated D-dimer level remained an independent predictor of poor functional outcome (OR 1.58 [95\% CI 1.19-2.09], $p=0.001$ ), after adjustment for the other covariates that were marginally or significantly associated with poor functional outcome in univariate analysis (Table 5).

Finally, to evaluate the impact of D-dimer on the accuracy of poorer functional outcome assessment, we compared the discriminatory abilities between models with and without D-dimer. In multivariable logistic regression analysis, increasing age (OR 1.05 [95\% CI 1.02-1.09], p $=0.001$ ) and poorer initial neurological status denoted by WFNS grade (OR 3.60 [95\% CI 1.44-9.04], $\mathrm{p}=0.006$ ) were also associated with poor functional outcome (Table 5). The $\mathrm{C}$ statistic for a model created by increasing age and WFNS grade was 0.829 and was significantly increased by addition of continuous D-dimer level (C statistic $=0.858, p=0.03)$. Furthermore, cross-tabulation for functional outcome between models with and without D-dimer levels is shown in Table 6. Among patients who had a poor functional outcome, 14 were correctly reclassified into a higher risk category, whereas 12 were inappropriately reclassified into a lower risk of poor outcome in the updated model with D-dimer levels. On the

TABLE 4. Univariate analysis of factors for poor functional outcome (mRS Score 3-6 at 3 months) after SAH

\begin{tabular}{lcccc}
\hline \multirow{2}{*}{ Variable } & \multicolumn{2}{c}{ Functional Outcome } & & \\
\cline { 2 - 3 } & Poor $(\mathrm{n}=91)$ & Favorable $(\mathrm{n}=96)$ & OR $(95 \% \mathrm{Cl})$ & $\mathrm{p}$ Value† \\
\hline Mean age \pm SD in yrs & $69.3 \pm 12.8$ & $59.4 \pm 14.1$ & $1.06(1.03-1.08)$ & $<0.001$ \\
\hline Women & $76(83.5)$ & $74(77.1)$ & $1.51(0.73-3.13)$ & 0.27 \\
\hline Hypertension & $32(35.2)$ & $51(53.1)$ & $0.48(0.27-0.86)$ & $\mathbf{0 . 0 1 4}$ \\
\hline Type 2 diabetes & $8(8.8)$ & $7(7.3)$ & $1.23(0.43-3.52)$ & 0.71 \\
\hline Antiplatelet/anticoagulant use & $10(11.0)$ & $2(2.1)$ & $5.80(1.24-27.3)$ & $\mathbf{0 . 0 2 6}$ \\
\hline WFNS Grades IV-V & $51(56.0)$ & $14(14.6)$ & $7.47(3.70-15.1)$ & $<0.001$ \\
\hline Posterior circulation & $15(16.5)$ & $18(18.8)$ & $1.70(0.95-3.05)$ & 0.69 \\
\hline Coil embolization & $47(51.6)$ & $37(38.5)$ & $2.43(0.61-9.62)$ & 0.07 \\
\hline Thick hematoma & $62(68.1)$ & $34(35.4)$ & $3.90(2.12-7.16)$ & $<0.001$ \\
\hline ICH & $22(24.2)$ & $9(9.4)$ & $3.08(1.33-7.12)$ & 0.008 \\
\hline IVH & $45(49.5)$ & $37(38.5)$ & $1.56(0.87-2.79)$ & 0.13 \\
\hline Median D-dimer in $\mu \mathrm{gg} / \mathrm{ml}$ (IQR) & $3.0(1.5-5.15)$ & $1.0(0.5-1.7)$ & $2.01(1.55-2.61)$ & $<0.001$ \\
\hline
\end{tabular}

* Values are presented as the number of patients (\%) unless indicated otherwise.

$\dagger$ Boldface type indicates statistical significance $(p<0.05)$ by univariate analysis. Italic type indicates marginally significant variables $(p<0.10)$. 
TABLE 5. Multivariable analysis of factors for poor functional outcome (mRS Score 3-6 at 3 months) after SAH

\begin{tabular}{lcc}
\hline \multicolumn{1}{c}{ Variable* $^{*}$} & Adjusted OR $(95 \% \mathrm{Cl})$ & p Value \\
\hline Increasing age & $1.05(1.02-1.09)$ & $\mathbf{0 . 0 0 1}$ \\
\hline WFNS Grades IV-V & $3.60(1.44-9.04)$ & $\mathbf{0 . 0 0 6}$ \\
\hline D-dimer & $1.58(1.19-2.09)$ & $\mathbf{0 . 0 0 1}$ \\
\hline
\end{tabular}

* Adjusted for hypertension, antiplatelet/anticoagulant use, coil embolization, thick hematoma, and ICH.

$\dagger$ Boldface type indicates statistical significance $(p<0.05)$ by multivariable analysis.

other hand, among patients who had a favorable outcome, 11 were correctly reclassified into a lower risk category, whereas 5 were inappropriately reclassified into a higher risk category in the updated model. The NRI and the IDI were calculated as $0.558(\mathrm{p}<0.001)$ and $0.057(\mathrm{p}<0.001)$, respectively, suggesting that $\mathrm{D}$-dimer has an additive predictive value for poor functional outcome on conventional risk factors.

\section{Discussion}

In this study, we characterized plasma D-dimer level on admission after aneurysmal SAH as being independently associated with systemic rather than neurological complications. In addition, D-dimer level augments the discrimination ability of our predictive model created by conventional risk factors for poor functional outcome.

D-dimer elevation is generally considered a stable parameter of hypercoagulation and subsequent fibrinolysis. ${ }^{2,14}$ In aneurysmal SAH, D-dimer levels correlate with other hemostatic parameters and reflect coagulopathic disturbances. ${ }^{14}$ The mechanism of D-dimer elevation after SAH remains unclear. Sudden elevations in intracranial pressure,,$^{10,18}$ tissue factor release via disrupted endothelium, ${ }^{6}$ dissolved subarachnoid clots entering cerebrospinal fluid circulation, ${ }^{8}$ and catecholamine surge, ${ }^{19}$ may trigger excessive thrombin generation and cross-linked fibrin turnover, leading to D-dimer elevation. In our study, increasing age was correlated with D-dimer elevations presumably because of physiological thrombophilia due to atherosclerotic change, increased comorbid burden, and decreased metabolism in elderly patients. ${ }^{16,24}$ In addition, D-dimer levels were significantly higher in women than in men in univariate analysis. This may be because the mean age of the female subjects $(66.3 \pm 14.0$ years) was older than that of the male subjects $(56.0 \pm 12.5$ years). Another possibility is an increased propensity of middle-aged or older women in our cohort to have thrombotic complications predisposing to D-dimer elevations, including venous thrombosis. ${ }^{12}$

Following aneurysmal rupture, elevated levels of Ddimer on admission are correlated with poor functional outcomes ${ }^{8,14,20}$ However, underlying mechanisms in which D-dimer affects functional outcomes have yet to be elucidated. In this study, we demonstrated that D-dimer elevation on admission was independently associated with increased incidence of systemic complications, including nosocomial infection, serum sodium disorder, and cardiopulmonary dysfunction. Patients with acute SAH possess increased risk of nosocomial infections secondary to a multitude of risk factors, for example, altered level of consciousness interfering with the ability to clear secretions, compromised host defenses, and presence of catheters and tubes, as well as decreased mobility while bed-ridden. ${ }^{7} \mathrm{Se}-$ rum sodium disorder is a well-characterized complication of SAH via the disrupted hypothalamic-pituitary axis, manifesting as syndrome of inappropriate secretion of antidiuretic hormone, salt wasting syndrome, or diabetes insipidus. ${ }^{23,28}$ Cardiopulmonary dysfunction emerges not only as specific complications after SAH, including Takotsubo cardiomyopathy and neurogenic pulmonary edema, ${ }^{15}$ but may also ensue if vasospasm prevention and treatment resulted in overhydration. It is possible that D-dimer elevation on admission may reflect ongoing microthrombi formation in the systemic microcirculation, thus causing dysfunction of multiple somatic systems, including the immune, endocrinological, and cardiovascular systems. In addition to acute SAH, D-dimer elevations are observed in chronic pathologies, such as hematological dyscrasias and malignancies. ${ }^{26}$ It is possible that subclinical states of these disorders predisposed patients to increased baseline D-dimer levels prior to their SAH ictus and resulted in poor functional outcomes.

In this study, multivariable analysis did not demonstrate D-dimer elevation at admission as a significant independent risk factor for neurological complications, including chronic hydrocephalus, DIND, or DCI. Previous studies demonstrated that D-dimer elevation was associated with neurological complications, including DIND and DCI., ${ }^{9,16,20}$

TABLE 6. Reclassification for the risk of poor functional outcome in aneurysmal SAH

\begin{tabular}{|c|c|c|c|c|c|c|c|c|c|c|}
\hline \multirow{3}{*}{$\begin{array}{c}\text { Basic } \\
\text { Model }^{*}\end{array}$} & \multicolumn{5}{|c|}{ Poor Outcome (mRS Score 3-6) } & \multicolumn{5}{|c|}{ Favorable Outcome (mRS Score 0-2) } \\
\hline & \multicolumn{4}{|c|}{ Basic Model + D-dimer } & \multirow[b]{2}{*}{ Total } & \multicolumn{4}{|c|}{ Basic Model + D-dimer } & \multirow[b]{2}{*}{ Total } \\
\hline & $<23 \%$ & $23-48 \%$ & $48-76 \%$ & $>76 \%$ & & $<23 \%$ & $23-48 \%$ & $48-76 \%$ & $>76 \%$ & \\
\hline$<23 \%$ & 3 & 2 & 0 & 1 & 6 & 38 & 3 & 0 & 0 & 41 \\
\hline $23-48 \%$ & 1 & 9 & 4 & 1 & 15 & 3 & 27 & 1 & 0 & 31 \\
\hline $48-76 \%$ & 0 & 6 & 15 & 6 & 27 & 0 & 7 & 12 & 1 & 20 \\
\hline$>76 \%$ & 0 & 0 & 5 & 38 & 43 & 0 & 0 & 1 & 3 & 4 \\
\hline Total & 4 & 17 & 24 & 46 & 91 & 41 & 37 & 14 & 4 & 96 \\
\hline
\end{tabular}

* The basic model includes age and WFNS grade. 
Yet, these studies were performed with univariate analyses alone, and confounding factors might not have been taken into account. Juvela et al. revealed that D-dimer level is a risk factor of DIND, independent of other known covariates by multivariable analysis. ${ }^{16}$ However, they measured D-dimer levels after surgical clipping, with open craniotomy procedures themselves increasing plasma D-dimer levels. ${ }^{9,20}$ As suggested by prior literature, prolonged elevations in D-dimer levels, rather than single-point measurements of D-dimer levels on admission, may lead to cerebral perfusion disturbances and emerging DIND. ${ }^{9,16}$ Secondary injury cascade propagated and aggravated by residual cerebral clot burden and procedure-related injury, rather than initial insult to the brain, may be involved in delayed ischemic complications.

Accurate outcome prediction after aneurysmal SAH remains challenging. The most popular grading system of initial clinical condition after SAH is the WFNS scale..$^{11,13}$ However, patients' neurological conditions fluctuate in the acute stages of SAH, and timing of grading is not clearly defined, leading to larger inter- or intraobserver variability. ${ }^{3,11}$ In addition, poor initial neurological grades (WFNS Grades IV and V) do not necessarily predict poor outcomes, whereby favorable outcomes were observed in $30 \%-50 \%$ of these patients. ${ }^{11,13,17}$ Owing to the association of D-dimer elevation with systemic complications, we make the assumption that $\mathrm{D}$-dimer elevation has additive predictive value on conventional risk factors, such as neurological grade and increasing age, predicting poor functional outcomes in a manner distinct from these traditional prognostic indicators. Together with ictal neurological insult and age-related decrease in brain plasticity, systemic complications associated with D-dimer elevation may lead to poor functional outcomes via aggravation of systemic metabolism, disturbances in cerebral perfusion, and alterations in brain oxygenation. Therefore, these systemic sequelae must be monitored and treated with vigilance in the neurocritical care unit, as many of these manifestations are recoverable.

Several limitations exist in our study. First, it is retrospective in nature and from a single institution. Findings should be interpreted cautiously, and potential interactions among variables may not be completely excluded by multivariable analyses. Second, no consensus is available regarding the normal ranges of D-dimer among different immunoassay kits of different institutions, which may hamper interpretation of results by future multicenter studies. ${ }^{916}$ However, our results suggest that plasma D-dimer level is a powerful objective tool to predict systemic complications and is associated with functional outcomes in a manner that is different from conventional risk factors after aneurysmal SAH. Further investigation is warranted to identify causative pathophysiological mechanisms of plasma D-dimer elevation to be associated with poor clinical outcomes, and to establish optimal treatment for SAH patients with high D-dimer levels.

\section{Conclusions}

Elevated plasma D-dimer levels on admission were independently associated with systemic complications after aneurysmal SAH. Thus, D-dimer elevation had an additive predictive value for poor functional outcome on conventional risk factors.

\section{References}

1. Arakawa Y, Kikuta K, Hojo M, Goto Y, Yamagata S, Nozaki $\mathrm{K}$, et al: Milrinone reduces cerebral vasospasm after subarachnoid hemorrhage of WFNS grade IV or V. Neurol Med Chir (Tokyo) 44:393-401, 2004

2. Barber M, Langhorne P, Rumley A, Lowe GDO, Stott DJ: Hemostatic function and progressing ischemic stroke: D-dimer predicts early clinical progression. Stroke 35:1421-1425, 2004

3. Chiang VL, Claus EB, Awad IA: Toward more rational prediction of outcome in patients with high-grade subarachnoid hemorrhage. Neurosurgery 46:28-36, 2000

4. DeLong ER, DeLong DM, Clarke-Pearson DL: Comparing the areas under two or more correlated receiver operating characteristic curves: a nonparametric approach. Biometrics 44:837-845, 1988

5. Drake CG: Report of World Federation of Neurological Surgeons Committee on a Universal Subarachnoid Hemorrhage Grading Scale. J Neurosurg 68:985-986, 1988 (Letter)

6. Findlay JM, Weir BKA, Kanamaru K, Espinosa F: Arterial wall changes in cerebral vasospasm. Neurosurgery 25:736746, 1989

7. Foreman PM, Chua M, Harrigan MR, Fisher III WS, Vyas NA, Lipsky RH, et al: Association of nosocomial infections with delayed cerebral ischemia in aneurysmal subarachnoid hemorrhage. J Neurosurg [epub ahead of print February 12, 2016. DOI: 10.3171/2015.10.JNS151959]

8. Fujii Y, Takeuchi S, Sasaki O, Minakawa T, Koike T, Tanaka R: Hemostasis in spontaneous subarachnoid hemorrhage. Neurosurgery 37:226-234, 1995

9. Fujii Y, Takeuchi S, Sasaki O, Minakawa T, Koike T, Tanaka $\mathrm{R}$ : Serial changes of hemostasis in aneurysmal subarachnoid hemorrhage with special reference to delayed ischemic neurological deficits. J Neurosurg 86:594-602, 1997

10. Fujii Y, Tanaka R, Takeuchi S, Koike T, Minakawa T, Sasaki $\mathrm{O}$ : Serial changes in hemostasis after intracranial surgery. Neurosurgery 35:26-33, 1994

11. Fung C, Inglin F, Murek M, Balmer M, Abu-Isa J, Z'Graggen WJ, et al: Reconsidering the logic of World Federation of Neurosurgical Societies grading in patients with severe subarachnoid hemorrhage. J Neurosurg 124:299-304, 2016

12. Heit JA, Spencer FA, White RH: The epidemiology of venous thromboembolism. J Thromb Thrombolysis 41:3-14, 2016

13. Hijdra A, van Gijn J, Nagelkerke NJ, Vermeulen M, van Crevel H: Prediction of delayed cerebral ischemia, rebleeding, and outcome after aneurysmal subarachnoid hemorrhage. Stroke 19:1250-1256, 1988

14. Ilveskero S, Juvela S, Siironen J, Lassila R: D-dimer predicts outcome after aneurysmal subarachnoid hemorrhage: no effect of thromboprophylaxis on coagulation activity. Neurosurgery 57:16-24, 2005

15. Inamasu J, Nakatsukasa M, Mayanagi K, Miyatake S, Sugimoto K, Hayashi T, et al: Subarachnoid hemorrhage complicated with neurogenic pulmonary edema and takotsubo-like cardiomyopathy. Neurol Med Chir (Tokyo) 52:49-55, 2012

16. Juvela S, Siironen J: D-dimer as an independent predictor for poor outcome after aneurysmal subarachnoid hemorrhage. Stroke 37:1451-1456, 2006

17. Mocco J, Ransom ER, Komotar RJ, Schmidt JM, Sciacca RR, Mayer SA, et al: Preoperative prediction of long-term outcome in poor-grade aneurysmal subarachnoid hemorrhage. Neurosurgery 59:529-538, 2006

18. Nakagomi T, Kassell NF, Sasaki T, Lehman RM, Fujiwara S: Etiology of the disruption in blood-arterial wall barrier fol- 
lowing experimental subarachnoid hemorrhage. Surg Neurol 34:16-26, 1990

19. Naredi S, Lambert G, Edén E, Zäll S, Runnerstam M, Rydenhag B, et al: Increased sympathetic nervous activity in patients with nontraumatic subarachnoid hemorrhage. Stroke 31:901-906, 2000

20. Peltonen S, Juvela S, Kaste M, Lassila R: Hemostasis and fibrinolysis activation after subarachnoid hemorrhage. J Neurosurg 87:207-214, 1997

21. Pencina MJ, D’Agostino RB Sr, D’Agostino RB Jr, Vasan RS: Evaluating the added predictive ability of a new marker: from area under the ROC curve to reclassification and beyond. Stat Med 27:157-172, 207-212, 2008

22. Pencina MJ, D’Agostino RB Sr, Steyerberg EW: Extensions of net reclassification improvement calculations to measure usefulness of new biomarkers. Stat Med 30:11-21, 2011

23. Qureshi AI, Suri MFK, Sung GY, Straw RN, Yahia AM, Saad M, et al: Prognostic significance of hypernatremia and hyponatremia among patients with aneurysmal subarachnoid hemorrhage. Neurosurgery 50:749-756, 2002

24. Righini M, Van Es J, Den Exter PL, Roy PM, Verschuren F, Ghuysen A, et al: Age-adjusted D-dimer cutoff levels to rule out pulmonary embolism: the ADJUST-PE study. JAMA 311:1117-1124, 2014

25. Sadamasa N, Yoshida K, Narumi O, Chin M, Yamagata S: Milrinone via lumbar subarachnoid catheter for vasospasm after aneurysmal subarachnoid hemorrhage. Neurocrit Care 21:470-475, 2014

26. Timp JF, Braekkan SK, Versteeg HH, Cannegieter SC: Epidemiology of cancer-associated venous thrombosis. Blood 122:1712-1723, 2013

27. von Elm E, Altman DG, Egger M, Pocock SJ, Gøtzsche PC, Vandenbroucke JP: The Strengthening the Reporting of Observational Studies in Epidemiology (STROBE) statement: guidelines for reporting observational studies. Lancet 370:1453-1457, 2007

28. Zheng B, Qiu Y, Jin H, Wang L, Chen X, Shi C, et al: A predictive value of hyponatremia for poor outcome and cerebral infarction in high-grade aneurysmal subarachnoid haemorrhage patients. J Neurol Neurosurg Psychiatry 82:213-217, 2011

\section{Disclosures}

The authors report no conflict of interest concerning the materials or methods used in this study or the findings specified in this paper.

\section{Author Contributions}

Conception and design: Fukuda, Lo. Acquisition of data: Fukuda, Yu Yamamoto, Handa, Yoshiharu Yamamoto, Kurosaki. Analysis and interpretation of data: Fukuda, Lo. Drafting the article: Fukuda, Lo. Critically revising the article: Fukuda, Lo. Reviewed submitted version of manuscript: Fukuda, Lo. Approved the final version of the manuscript on behalf of all authors: Fukuda. Statistical analysis: Fukuda, Lo, Yoshiharu Yamamoto. Administrative/technical/material support: Yu Yamamoto, Handa, Yoshiharu Yamamoto, Kurosaki. Study supervision: Yamagata.

\section{Correspondence}

Hitoshi Fukuda, Department of Neurosurgery, Kurashiki Central Hospital, 1-1-1, Miwa, Kurashiki City, Okayama 710-0052, Japan. email: fukudaharpseal@gmail.com. 